\title{
A Dolphin Is a Dolphin Is a Dolphin? Multimedia Enriched Learning Objects in NEMO
}

\author{
Benjamin Feldner, Silke Günther, Felix Schmitt, Thomas Winkler, Michael Herczeg \\ Institute for Multimedia and Interactive Systems - University Lübeck \\ feldner|guenther|schmitt|winkler|herczeg@imis.uni-luebeck.de
}

\begin{abstract}
In this paper we present a concept for integrating Multimedia-Enriched Learning Objects (MELOs) within a unifying technological framework for schools. First, we discuss the general role of objects for child development and learning. We then outline the framework architecture of the proposed Networked Environment for Multimedia Objects (NEMO). Afterwards, we give examples to describe how the use of MELOs could enhance the learning experience of primary school children while learning about dolphins and discuss the pedagogical benefits of our approach.
\end{abstract}

\section{Learning and (digital) objects}

Objects can serve many different activities, such as observing, carrying, creating and exchanging, which turns them into powerful learning mediators in our world. Object permanence and persistence are important cognitive concepts for children. While learning to differentiate between inside and outside, children use transitional objects, e.g. teddy bears or blankets [1]. Even these two simple examples underline the importance and the complexity of object-related learning.

Since its introduction in the mid 1990s, the term Learning Object (LO) has referred to all kinds of educational material, leading to a multitude of definitions and uses. Creating, aggregating and retrieving content is an essential part of computer-mediated learning, but learning processes are inextricably linked to the characteristics of the individual learners, the context and the chosen media. Thus, like any other object, a LO can be assimilated in many different ways, e.g. depending on its form, function and the derived meanings $[2,3]$.

\section{Dolphins as objects of learning}

Choosing and using a medium as a setting for teaching and learning imposes certain restrictions on educators, learners and learning objects, which can be partially circumvented by innovative methods and didactical approaches.

Learning about marine mammals in a projectoriented way is part of the curriculum for $4^{\text {th }}$ graders in German elementary schools. Becoming knowledgeable about dolphins is not supposed to be about reciting facts, such as that there are almost forty species of dolphin in seventeen genera, which can be found all over the world. Instead, the teachers are encouraged to aid the children in experiencing dolphins as intertwined with highly complex issues like the destruction of ecosystems and food chains.

Being objects of learning, dolphins are presented and created in various forms and functions. Therefore they are not only examples of sea mammals, but also illustrate the complexities of learning processes.

\section{Educational multimedia systems}

In the light of these general reflections on objects used to support learning, two multimedia systems are presented with regard to different educational settings.

Moodle [4] is an example of an open source Learning Management System (LMS) to enable webbased e-learning. The Moodle environment provides a wide range of communication tools and joint online access to multimedia educational resources. As a course-based online learning platform, supplemented by features for conducting surveys and tests, Moodle familiarizes teachers with the concept of sharing courses and learning scenarios that have proven to be successful in e-learning, but it does not equip them and their students with means of enriching learning objects in multiple modes and media once they have been created. 
Designed as an interactive augmented reality environment for individual as well as collaborative learning, ARiSE [5] aids students in utilizing learning objects augmented with 3D graphics. Being a networked learning system, ARiSE does not restrict computer-supported education to a single location at a time. The new system NEMO (Networked Environment for Multimedia Objects) sketched out in this paper is intended to support the multimedia enrichment of objects as well as the acquisition of media literacy that is not based on a secluded understanding of media like visual literacy or print literacy imply. Instead, it is rooted in an evolving understanding of the interplay between different computer systems and their overarching as well as distinguishing features with regard to learning processes. The cornerstones of the intended technical realization are incorporated into a unifying architectural concept presented in the following sections.

\section{Technical realization}

The core of the implementation of NEMO is an integrated architecture for a distributed system based on a client-server model, enabling the students to explore different modes of enriching learning objects while interacting with the system.

The architectural concept includes three elements that are described in the following subsections.

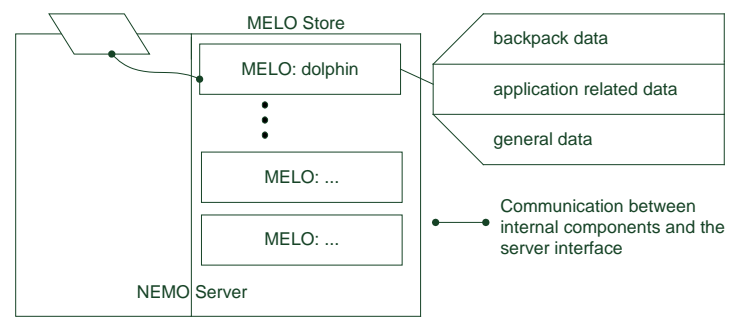

Figure 1. Structure of the NEMO Server

\subsection{Composition of the MELOs}

The MELOs (Multimedia-Enriched Learning Objects) carry three independent sets of data that can be used in separate contexts of the system (cf. Figure 1).

Irrespective of the client application, there is a set of general data, such as the creation date or ownership of the MELOs which is an elementary part of this data set. For interchangeability with other e-learning environments information about the MELO can also be stored in the general data set using an appropriate metadata standard, such as the LOM data model [6].

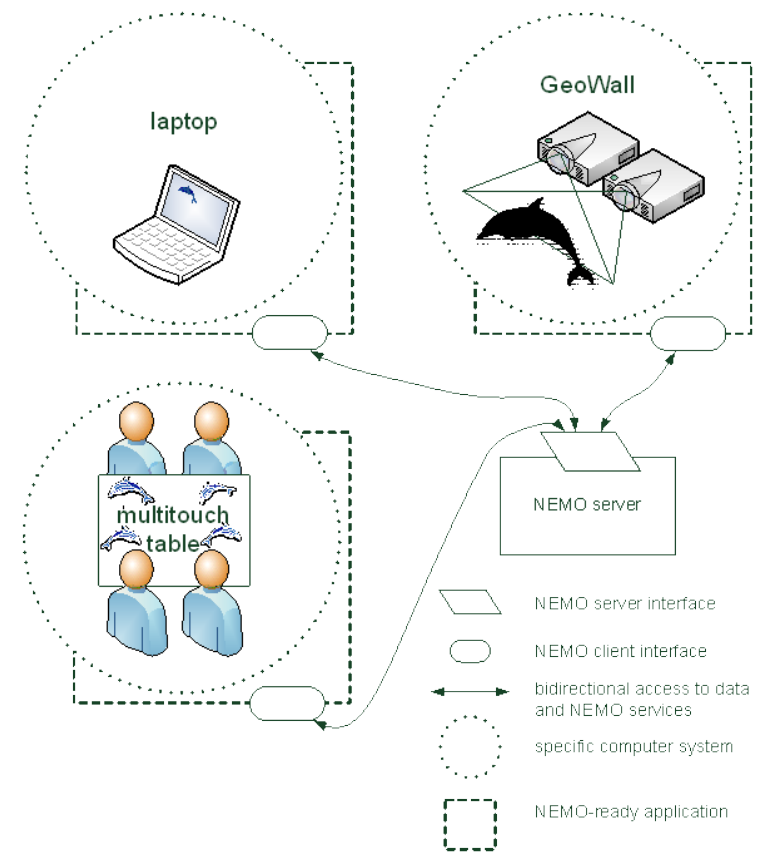

Figure 2. Accessing and using MELOs

Client-specific data refers to the technological specifications of enriching the MELOs, e.g. data required to render a 3D-dolphin or data to position the dolphin in relation to other mammals on the multitouch table.

Backpack data supports students and teachers in understanding and utilizing enrichment processes that have taken place while interacting with the system. In addition, teachers and students can use the backpack data collected by the system to trace and retrace media and interaction paths while reflecting on their learning strategies.

\subsection{Client applications}

Figure 2 illustrates the use of a dolphin object on three different computer systems. The respective software applications make use of a unified interface to access services provided by the NEMO server. Since the interface is bidirectional, the server can also access data offered by the client application.

\subsection{The NEMO server}

The server stores and manages MELOs in a database and provides a client interface for accessing MELO data and linked resources, synchronous and asynchronous communication between NEMO applications 
and access to information about which MELOs are in use by other applications.

\section{A scenario for using MELOs}

The exemplified scenario for project-oriented work on dolphins involves $4^{\text {th }}$ graders, aged 9-11, at a local elementary school. The students have access to laptop computers, a multitouch table, a 3D presentation wall (like the GeoWall [7]) and MOLES, the Mobile Learning Exploration System developed at our institute [8].

For each student a dolphin object is created in the system functioning as an exemplary MELO. Additionally, there is a dolphin object that belongs to the whole class and is used for presentations and usage instructions for the system. At the beginning of the learning unit, the teacher presents a virtual dolphin on the $3 \mathrm{D}$ projection screen. Basic facts about dolphins and related topics are discussed in the class. The dolphin can be rotated and its typical movements like breathing, jumping, or swimming can be animated. Afterwards, the students use the NEMO system with a laptop computer connected to the Internet to research facts for their personal dolphin object: Where do dolphins live? What sounds do they make? After the personal MELOs have been enriched, they are pushed to the multitouch table and shown on a world map. The collected detailed information can be displayed on the map and sounds can be played. To test the transfer of dolphin-related knowledge, the teacher may link questions to the dolphin objects which the children can answer, e.g. with their mobile phones.

In the end they can "store" their personal dolphin on their own mobile device (a kind of personal MELO wallet) to take it with them. Actually, they only store the access information and signature of their personal learning objects which resides on the server.

\section{Conclusion}

MELOs can facilitate learning at different levels of complexity. The perceived level of complexity depends on the task itself and the learners' competencies. When perceiving a high level of complexity, students associate the task at hand with a lack of transparency and limited influence capabilities, leading to behavioral patterns, such as oversimplification or denial, which inhibit successful learning processes. Complexity can be conquered by reducing intricate systems and situations to simpler ones, but there is no meaningful learning without at least getting a basic understanding of the bigger picture. With MELOs, the interdepen- dencies within a complex multimedia system become scalable more easily.

NEMO is a multimedia learning system that enables students to choose their own media paths while exploring and enriching digital objects. Instead of being passive recipients of new technologies, students develop higher level skills by rearranging and reintegrating learning material. Moreover, the internal differentiation of learner groups is supported by facilitating learning at different levels of complexity, flexibility with regard to time-on-task, and the acquisition and refinement of learning strategies. These anticipated educational advantages are furnished by the integrated architecture for a distributed system, which consists of client applications, the NEMO server, and the MELOs. The independent sets of data carried by the MELOs ensure flexibility and reusability of the learning material. Providing students with objects they can enrich within the realms of different media opens up multiple learning strategies for objects and their intertwinements.

\section{References}

[1] D. Winnicott, "Transitional Objects and Transitional Phenomena-A Study of the First Not-Me Possession,” International Journal of Psycho-Analysis, vol. 34, pp. 89-97, 1953.

[2] P. Polsani, "Use and Abuse of Reusable Learning Objects," Journal of Digital Information, vol. 3, no. 4, 2003.

[3] N. Friesen, "Three objections to learning objects and e-learning standards," Online education using learning objects, pp. 59-70, 2004.

[4] J. Cole, "Using moodle." O'Reilly, 2005.

[5] M. Bogen, J. Wind, and A. Giuliano, "ARiSEAugmented Reality in School Environments," LECTURE NOTES IN COMPUTER SCIENCE, vol. 4227, pp. 709-714, 2006.

[6] L. Standard, "Draft Standard for Learning Object Metadata, IEEE P1484. 12/D4. 0,” Disponible: http://ltsc.ieee.org/doc/wg12/LOM_Wd4.doc.Consulta. [7] A. Johnson, J. Leigh, P. Morin, and P. Van Keken, "GeoWall: Stereoscopic Visualization for Geoscience Research and Education," IEEE COMPUTER GRAPHICS AND APPLICATIONS, pp. 10-14, 2006.

[8] S. Günther, T. Winkler, and M. Herczeg, "Mobile Learning with Moles: A Case Study for Enriching Cognitive Learning by Collaborative Learning in Real World Contexts," In J. Luca and E.R. Weippl (Eds.) Proceedings of ED-MEDIA 2008. Chesapeake/VA: AACE. pp. 374-380, 2008. 